Patient perception, preferences and participation

\title{
Time intervals in diagnosing Parkinson's disease: The patients' views
}

\author{
Annette O.A. Plouvier ${ }^{\mathrm{a}, *}$, Tim C. Olde Hartman ${ }^{\mathrm{a}}$, Leontien P.W. Boots ${ }^{\mathrm{a}}$, Bastiaan R. Bloem ${ }^{\mathrm{b}}$, \\ Chris van Weel ${ }^{\mathrm{a}, \mathrm{c}}$, Antoine L.M. Lagro-Janssen ${ }^{\mathrm{a}}$ \\ ${ }^{a}$ Department of Primary and Community Care, Radboud university medical center Nijmegen, The Netherlands \\ ${ }^{\mathrm{b}}$ Department of Neurology and Parkinson Centre Nijmegen, Donders Institute for Brain, Cognition and Behaviour, Radboud university medical center \\ Nijmegen, The Netherlands \\ ${ }^{\mathrm{c}}$ Australian Primary Health Care Research Institute, Australian National University, Canberra, Australia
}

\section{A R T I C L E I N F O}

\section{Article history:}

Received 4 September 2014

Received in revised form 22 December 2014

Accepted 7 February 2015

\section{Keywords:}

Parkinson's disease

Diagnostic pathway

Patient's perspective

Qualitative research

\begin{abstract}
A B S T R A C T
Objective: To explore patients' views on their pathway to the diagnosis of Parkinson's disease (PD). Methods: A qualitative study of 52 essays written by patients with PD, using comparative content analysis.

Results: Patients divide their diagnostic pathway into three time intervals: recognition of the symptoms; the decision to seek help; and the process of diagnosing PD. Patients have difficulties recognizing the prodromal symptoms of PD (their knowledge is based on public figures with advanced PD) and mention their general practitioners do as well. The decision to seek help is influenced by the patient's attitude toward health care and by their significant others. More than half of the patients believe their diagnosis was delayed. However, the majority of all patients are satisfied with their diagnostic trajectory.

Conclusion: The pathway to diagnosing PD is an iterative process influenced by patient-, health care provider- and disease-related factors. Despite possible delay in diagnosis, patients are content with their pathway.

Practice implications: In order to facilitate earlier diagnosis and timely therapeutic intervention (in particular with regard to future possibilities for disease modifying therapy), effort is required to increase the recognition of prodromal symptoms of PD by patients, their significant others and health care providers.
\end{abstract}

(c) 2015 Elsevier Ireland Ltd. All rights reserved.

\section{Introduction}

Parkinson's disease (PD) is now recognized as a multisystem disorder with motor and non-motor features [1]. Some motor and non-motor features are prodromal symptoms: symptoms that are already present before the onset of the typical motor signs of PD [2]. Patients seem to have prodromal symptoms years before they are diagnosed with PD [2-4]. As physical abnormalities do not immediately lead to symptoms, the process is influenced by attention, awareness, interpretation and attribution of the patient $[5,6]$. The illness representations, which patients form of a perceived health threat, influence coping strategies including help seeking behavior $[7,8]$. Earlier research has shown that it takes

\footnotetext{
* Corresponding author at: Department of Primary and Community Care, Radboud university medical center, Postbus 9101, 6500 HB Nijmegen, The Netherlands. Tel.:+31 0243668036.

E-mail address: Annette.Plouvier@radboudumc.nl (Annette O.A. Plouvier).
}

patients more time to recognize their motor symptoms and to realize they need medical attention, than it takes the general practitioner (GP) to diagnose PD [9]. On the other hand, it is not uncommon that a patient needs to visit a number of health care providers before the diagnosis of PD is made [10]. When it comes to women and patients with young onset PD, health care providers seem to require more time to diagnose PD [11,12]. However, patients can benefit a lot from an early diagnosis of PD. Early recognition of symptoms allows patients and health care providers to discuss the benefits of timely therapeutic intervention on the one hand and the risk of side effects on the other. They can then make a shared decision on a customized balance between advantages and disadvantages, taking into account the patient's personal situation and preferences. This is likely to result in maintenance of quality of life, slowdown of clinical progression and reduced mortality [13-15].

Research in patients with cancer has shown that the pathway from the first recognizable symptoms to the diagnosis can be influenced by a number of factors such as the nature of the 
symptoms and the emotional response to them, knowledge of the disease and the consultation of significant others [16]. In chronic diseases with a less threatening outcome, such as diabetes mellitus, the same factors are of importance [17]. For PD it is unknown which factors influence the diagnostic pathway and how patients reflect on their pathway. However, more insight into the patients' views could lead to interventions that facilitate an earlier diagnosis by avoiding as much delay as possible in the diagnostic pathway. In this study we aim to gain insight into the patients' views on their diagnostic pathway and the factors that influence it. Furthermore, we want to know how patients reflect on their pathway.

\section{Methods}

\subsection{Recruitment}

This study is part of a larger study on the prodromal symptoms of PD and the patients' views on the trajectory toward the diagnosis. For this reason, all patient members of the Dutch Parkinson's Disease Association whose email addresses were known $(n=4717)$, received an email, in which the study was explained and they were asked to participate. In case patients were willing to participate a digital essay format was sent. Digital essays rather than individual interviews were chosen to assure easily accessible, anonymous participation and to enable patients to reflect in their own pace. Participation was voluntarily and onetime only. Patients were provided with contact information in case of questions, concerns or hesitations about participation. After completion of the essay format, patients had to agree with submission of the format. This step was assessed as Informed consent.

\subsection{Data collection}

Patients were asked for their demographic characteristics at the time of diagnosis: sex, age, level of education, employment status and civil status. To help them formulate their essay, a number of questions, based on literature and expert opinion, were developed in a small pilot study. The final questions are shown in Table 1.

Of all the patients who received an email, $27 \%(n=1251)$ started writing an essay. Essays were completed by $21 \%(n=974)$ of the patients: 689 patients responded before the reminder, 285 afterwards. Patients with a different diagnosis than idiopathic PD were excluded $(n=74)$. Finally 900 essays remained (Fig. 1$)$.

\subsection{Data analysis}

A purposive sample of 26 essays was entered into ATLAS.ti 7, a software program for detailed coding in qualitative data analysis. Purposive sampling, based on the collected demographic data, was used to increase the external validity and to provide a wide range

Table 1

Subject questions included in digital essay format.

\begin{tabular}{ll}
\hline Question & \\
\hline 1 & $\begin{array}{l}\text { Can you describe your first complaint(s) that eventually turned } \\
\text { out to be a forerunner sign of PD? What did you do when you } \\
\text { experienced this/these complaint(s)? }\end{array}$ \\
& $\begin{array}{l}\text { Can you describe what happened next, until the moment you } \\
\text { were diagnosed with PD? }\end{array}$ \\
& $\begin{array}{l}\text { Did people in your surroundings influence the pathway } \\
\text { to diagnosis? If so, in what way? } \\
\text { Do you think, in your case, it would have been possible to } \\
\text { diagnose PD earlier? If so, at what time and why do you think so? } \\
\text { What were the consequences for you and your family? }\end{array}$ \\
\end{tabular}

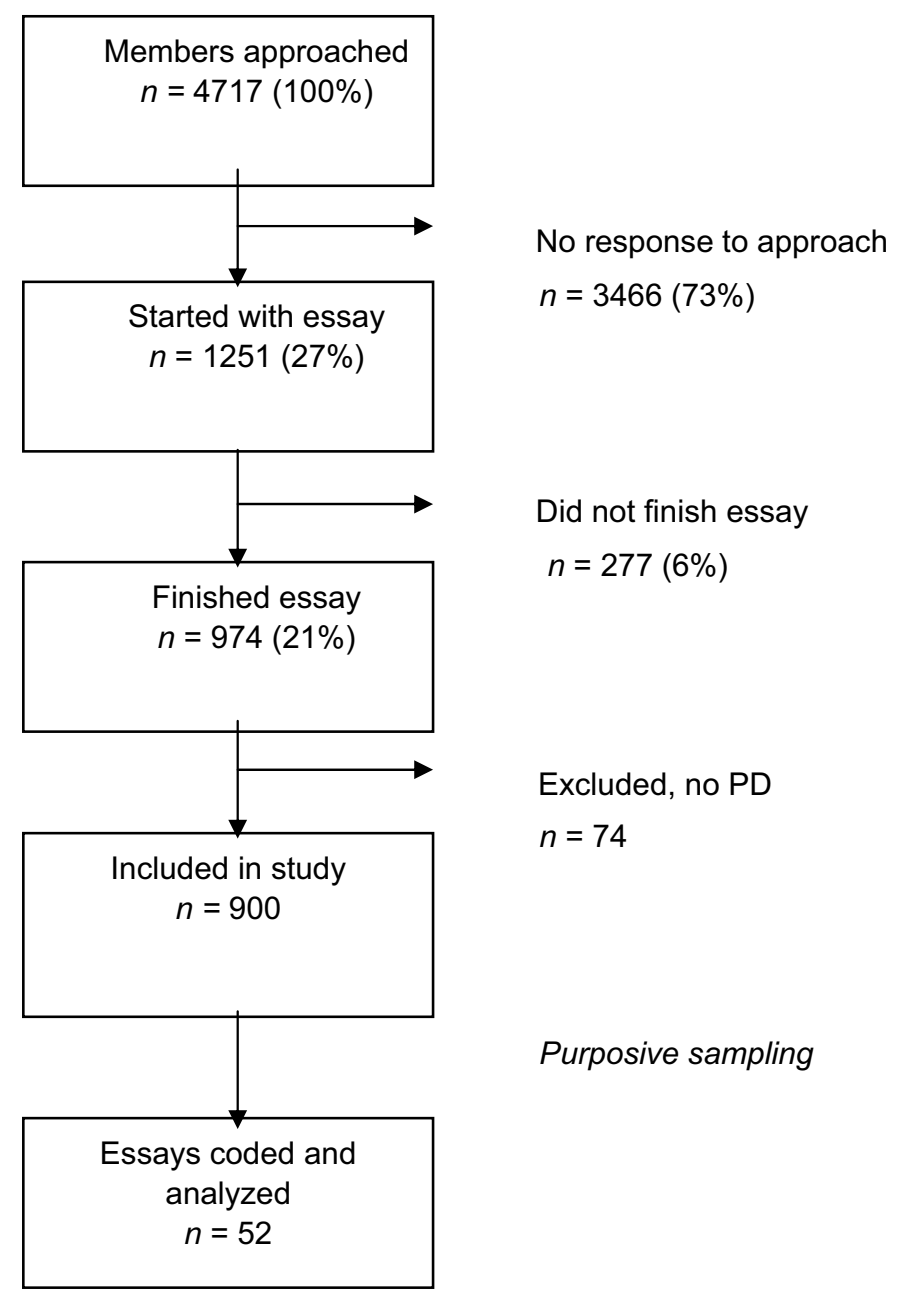

Fig. 1. Selection of essays

of patients' experiences. Level of education was divided into two categories: low and high. Finishing elementary school or vocational education was considered a low level of education. Finishing high school, higher professional education or university was considered a high level. The qualitative data analysis was an iterative process by two independent researchers (AP, LB), using the principles of comparative content analysis [18]. The two researchers read the 26 essays several times to familiarize themselves with the data. They independently applied codes to meaningful words and sentences in the essays. These codes were discussed and refined in consensus meetings with the supervisors (TOH, AL). New codes, arising from the discussion, were applied to the essays. After analysis of the 26 essays no significant new codes emerged (saturation). Codes were then grouped into themes, final themes were agreed upon with the supervisors (TOH, AL). These themes were structured in Fig. 2. In order to verify the figure and the position of the themes within it, another purposive sample of 26 essays was analyzed.

\section{Results}

\subsection{Characteristics of the study population}

About half of the participants were women (54\%) (Table 2).The mean age at the time of diagnosis was 56 years (SD 14.5), varying between 32 and 84 years. The majority of the patients was employed (62\%) and lived with a partner and/or children (75\%) at the time of diagnosis. 


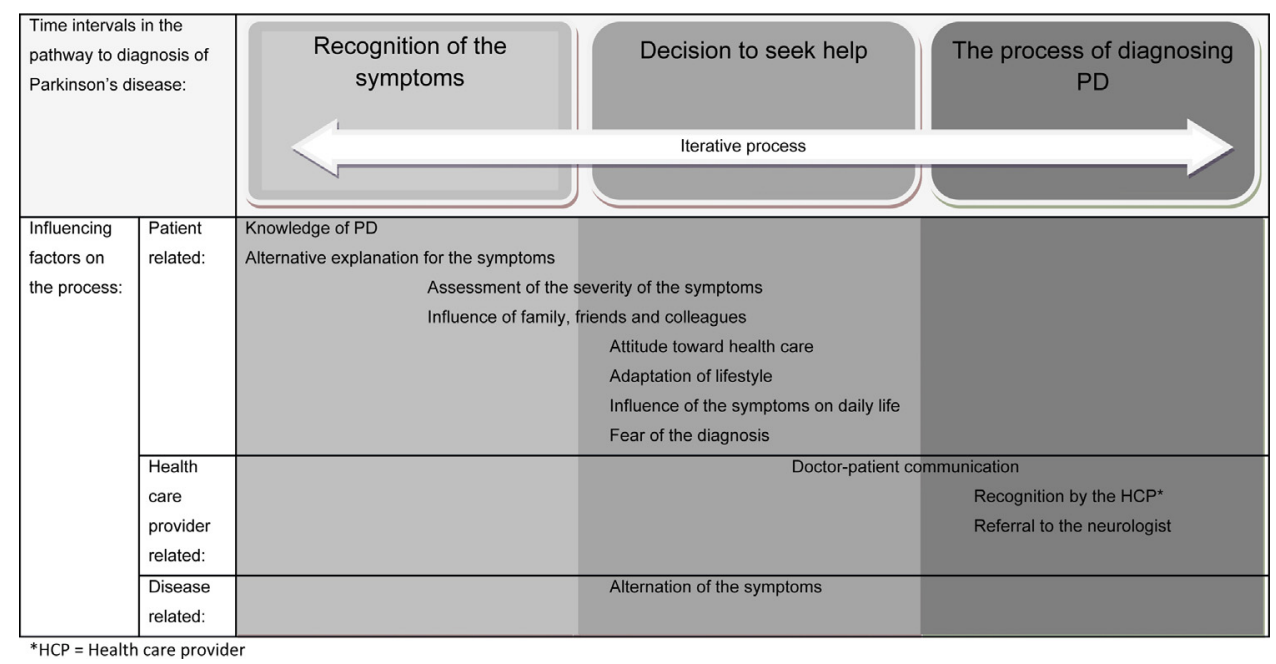

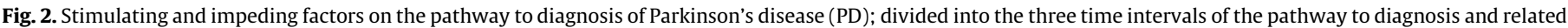
to factors concerning the patient, the health care provider and the disease. ${ }^{*} \mathrm{HCP}=$ health care provider.

\subsection{Diagnostic process}

We could distinguish three time intervals in the individual pathways to diagnosis, as described by patients: recognition of the symptoms, the decision to seek help from health care providers and the process of diagnosing PD (Fig. 2). Each of these intervals will be further explored beneath.

\subsubsection{Recognition of the symptoms}

Many patients stressed the importance of recognizing the first symptoms. The majority did not immediately recognize that their symptoms could be part of a disease. Rather, they described they realized something was abnormal. Family, friends or colleagues were described to be of influence in the recognition.

Back from holiday, I thought that the handlebar of my folding bike was loose-it wobbled quite a bit. Perhaps a few days later, I thought the wheel of my regular bicycle wobbled as well. This could not be a coincidence, it had to be me (Female, 53 years).

[...] I took part in a walking event that spanned four evenings. One of my friends noticed my right arm did not swing while I was walking (Female, 32 years).

Table 2

Sample characteristics at the time of diagnosing Parkinson's disease.

\begin{tabular}{llr}
\hline Demographic variable & & $n=52$ \\
\hline Sex & Male & 24 \\
& Female & 28 \\
Mean age in years (SD) & $56.3(14.5)$ & \\
Level of education & High & 26 \\
& Low & 26 \\
Employment & Employee & 25 \\
& Self-employed & 2 \\
& Retired & 12 \\
& Receive sickness benefit & 4 \\
& Unemployed & 4 \\
& Combination of employments & 5 \\
Civil status & With partner/family & 35 \\
& Single* & 13 \\
& Single with children & 4 \\
\hline
\end{tabular}

\footnotetext{
Including widowed and divorced.
}

Many patients described they initially found an alternative explanation for their symptoms, sometimes influenced by previous experiences or by family and friends.

I experienced difficulties with writing, which I noticed when writing many Christmas cards in December [...] I blamed increased computer use being the reason I was no longer accustomed to handwriting a lot (Female, 47 years).

I had some difficulties washing my hair. The right side was slower than the left side. When I said something about it, my children responded: mother you are getting older (Female, 54 years).

A few patients mentioned that they only knew what advanced PD looked like, based on information from books or television. That was the reason they had not taken PD into consideration.

For me at that time, the image of Parkinson's disease was defined by Prince Claus [member of the Dutch royal family] and the Pope [John Paul II], not knowing they already had an advanced stage of Parkinson's disease (Female, 47 years).

However, a few patients instantly identified their symptoms as a disease or even as PD.

In February [...] my leg started to shake and I knew I had Parkinson's disease (my sister has it too and an acquaintance as well) (Female, 68 years).

\subsubsection{Decision to seek help from health care providers}

After recognizing that the symptoms were abnormal, patients indicated that they needed to make a decision whether to consult their GP or not. Several patients decided to seek help right after detecting the symptom(s), sometimes stimulated by family, friends or colleagues. However, a few patients seemed to have difficulties assessing the severity of their symptoms. They mentioned they were afraid what the health care provider might think of them and feared that their symptoms were due to dramatizing, or that they were wasting the health care provider's time.

I visited the GP for a diagnosis when the thought occurred to me: there is really something physically wrong with me, this is not all in my head or the consequence of being alone with two kids making everything difficult, difficult, difficult (Female, 49 years). 
I remember that I felt guilty for taking his time, and I felt uncomfortable in the waiting room among the 'real patients' (Female, 47 years)

The patient's attitude toward health care appeared to be of importance. Patients think differently about consulting health care providers. Previous negative experiences in the communication with health care providers might influence the decision to return to the doctor with symptoms.

I grew up in an environment that favors alternative medicine, and thus did not seek help from mainstream medicine until later on (Female, 70 years).

[...] Pain in my right leg and the lower back. Physical therapy. [...] Still tired. Sometimes a severe cramping around my heart. [...] In June [...] extensive testing, diagnosis of angina pectoris. Walking becomes more difficult: dizziness, my handwriting becomes spidery. Now and then blood tests, the results are always normal. My GP: "There is nothing wrong with you." But where does this fatigue come from then? GP: "Well, I'm not sure. But you're not that young anymore!" I felt really tired lately, especially in the morning when getting out of bed. I get depressed (Female, 84 years).

Some patients described they adapted their lifestyle or made adjustments in order to relieve the symptoms.

My left hand started to shake in more and more situations, it was especially bad in the choir; new sheet music in my hands, reading music and lyrics, and singing at the same time. [...] At the December concert, I sang with a home-made brace to control my hand (Female, 53 years).

Patients indicated that they altered their decision to seek help when the symptoms got worse, new symptoms appeared or symptoms did not recover spontaneously. The restrictive influence of symptoms on the patient's daily life was also mentioned as a reason to visit a health care provider.

After a good holiday with my family, the tremor still continued, so I visited the GP again (Male, 32 years).

At one point, I became tired very easily, I lost strength in my arms. I was a production worker and noticed I had more and more trouble with my motor skills, then I went to the GP (Male, 42 years).

A few patients actively decided not to seek help yet, because of fear for the diagnosis.

The first symptom was a tremor of the right hand which came about suddenly. Furthermore, my husband and friends noticed that I did everything slower. For me, the worst part was the feelings of depression; that was not my nature. I had everything I ever wanted, but I could not enjoy it, it was horrible. My handwriting had changed as well, it became very small. I struggled with it for two years, but deep in my heart I knew it was not good and that I probably had Parkinson's disease (Female, 61 years).

\subsubsection{The process of diagnosing $P D$}

Some patients described the GP immediately noticed that their symptoms could be signs of PD and referred them to the neurologist, who instantly diagnosed the disease. However, more than half of the patients mentioned their GP did not recognize the symptoms, sometimes not even when the patient specifically asked if it could signal PD. Other patients described they were referred to the physiotherapist or orthopedic surgeon without a clarified diagnosis. Some patients mention their neurologist seemed to have difficulties recognizing the symptoms.

My fingers no longer cooperated. I could not hold a pen and had constipation. Then I visited the GP. The GP did not recognize it as PD. He said the problems were caused by aging (Male, 69 years).

I had shoulder complaints. I went to the GP, who referred me to the orthopedic surgeon. He told me I had bursitis which was treated by injections in my shoulder. I had to come back every 6 weeks [... . I I was treated for 2 years like this (Male, 52 years).

\subsection{Reflecting on the pathway}

Patients reflected differently on their diagnostic pathway. Nearly half of the patients believed they could not have been diagnosed earlier.

I do not think I could have been diagnosed faster. My complaints are always taken seriously, even when they were 'vague'. Some can, retrospectively, be attributed to PD (Male, 83 years).

However, the majority of the patients did believe their diagnosis was delayed. A few patients felt this was the result of their own help-seeking behavior. Others described that they believed the health care provider did not recognize their symptoms in time or postponed referral to a neurologist. Nevertheless, most patients were satisfied with the trajectory toward diagnosis. A few discontent patients described difficulties in the communication with their doctor.

If I look back, then the diagnosis probably could have been made earlier, but this was my doing. But I do not regret the path I took and that I only took action after 2 years (Female, 61 years).

If I had gone to the GP earlier, and the GP had known more about $\mathrm{PD}$, then some things could have been detected faster. If the diagnosis was made earlier, I could have felt better about it emotionally (Male, 53 years).

\section{Discussion and conclusion}

\subsection{Discussion}

To the best of our knowledge, this is the first study that explores the pathway to the diagnosis of PD from the patients' viewpoint. The pathway to the diagnosis of PD, as described by patients, can be divided into three time intervals: recognition of the symptoms, the decision to seek help and the process of diagnosing PD. Impeding and stimulating factors concerning the patient, the health care provider and the disease itself can influence each of these time intervals. Although more than half of the patients believed their diagnosis was delayed, the majority of all patients were satisfied with the trajectory toward diagnosis.

Earlier studies have shown that the pathway to the diagnosis of a disease can be divided into several stages in which delay can appear. Safer et al. proposed a model of three stages of delay in seeking care at a medical clinic [19]. Andersen et al. and Walter et al. have built further on this, resulting in the model of Walter that contains four intervals with clearly defined start and endpoints: the appraisal interval (from detection of bodily change(s) to perceiving a reason to discuss symptoms with a GP); the helpseeking interval (from perceiving a reason to visit the GP to the first 
consultation with a GP); the diagnostic interval (from the first appointment to the diagnosis); and the pre-treatment interval (the time between the diagnosis and initiation of treatment) $[20,21]$. With the exception of the pre-treatment interval, this model seems well applicable to our results.

According to the model of Walter et al. [21], the intervals of the pathway to diagnosis are influenced by patient-, health care provider- and disease-related factors. Most influencing factors found in our study are patient-related and can be stimulating as well as impeding. These include (lack of) knowledge of PD, alternative explanations for the symptoms, assessment of the severity of the symptoms (and possible interpretation as common illnesses), the influence of family, friends and colleagues, adaptations in lifestyle to relieve the hinder and the restrictive influence on daily life. These results are in line with the factors found to be of influence in studies on cancer and diabetes [16,17,22]. However, our study reveals that the media play an important role as well on the diagnostic pathway of PD. Books and television paint a classic picture of $\mathrm{PD}$, thereby limiting the knowledge of the disease and influencing the assessment of severity. Furthermore, our findings reveal that fear of the diagnosis can hold-back patients from seeking help. Although this fear is described in cancer research as well, fear for cancer might be difficult to compare to fear for PD. Patients associate cancer with painful treatments and death [22] while they might not have such explicit ideas about PD. Since most prodromal symptoms of PD are not acute or life threatening, patients can decide to postpone seeking help. Our study showed that the patient's attitude toward health care providers, sometimes prompted by earlier experiences, can also influence the decision to seek help. Some patients mention they are hesitant to present their non-specific symptoms to their GP, afraid they might be seen as somatizers. This is in line with earlier research that showed that patients carefully consider when to consult their GP and are concerned about going with non-specific symptoms $[16,17,22,23]$. Research in epilepsy also showed that patients might postpone seeking help because they are not ready to accept the diagnosis of a chronic disease [24]. The same might be true for patients with PD.

A number of health care provider-related factors influence the diagnostic pathway as well. Health care providers need to recognize the (prodromal) symptoms and suspect PD. The importance of educating physicians to consider the possibility of prodromal PD is already expressed in earlier research [25]. However, communication also seems to be an important factor influencing patients' contentment with their diagnostic pathway [26]. It is desirable that patients are provided with customized information concerning the suspected diagnosis and treatment options. They should be encouraged to participate in the decision making process on referral to a movement disorder specialist and on therapy [14,27]. For this, physicians need to persuade patients to ask questions, articulate their expectations and voice their preferences [28].

Finally, disease-related factors are of influence on the diagnostic pathway, in particular on the decision to seek help. In line with literature $[16,17,22]$, we found that patients are more inclined to seek help when their symptoms become worse or do not recover spontaneously. However, this requires patients who are aware of bodily changes and are capable to carefully monitor their symptoms [19]. In addition, the general population should be more aware of symptoms, that can be highly relevant for the early detection of PD [25].

The pathway to diagnosis is a dynamic and an iterative process in which patients may not experience a linear passage through the intervals, and in which steps can be repeated until PD is diagnosed. This is also shown in the model of Walter et al. [21]. Based on that model we developed a figure that summarizes all the influencing factors found in this study, as discussed above (Fig. 2).
Asking patients to retrospectively describe their diagnostic pathway inevitably leads to limitations. Patients may find it difficult to remember precisely what took place prior to the diagnosis, at what time and in which order, especially since for some patients it is years ago since they were diagnosed. Therefore, recollection bias cannot be ruled out. However, with the use of a digital essay format we gave patients the opportunity to go back in time and recall the pathway, while supporting the arrangement of their memories. The format with open questions led to essays of comparable structure, at the same time maintaining the possibility for the patients to individualize their answers. Unfortunately, the time frame in the essays is not always clear. Therefore there are limitations in comparing the time frame of each interval with other studies $[9,26]$.

The preset questions and the approach via internet have other disadvantages. The extent of details found in the essays is limited; in depth interviews could have given more detailed information on the diagnostic pathway. Moreover, it is estimated that less than $25 \%$ of all patients with PD in The Netherlands is a member of the Dutch Parkinson's Disease Association. Furthermore, only a selection of the patient members of the Association is reached with the approach through email and the use of a digital essay format. Members who are unable to use a computer or feel uncomfortable with it are left out. Although information on the demographic variables of the non-responders and the reasons why they did not take part is lacking, the inability or undesirability to use the computer may be the explanation that the respondents, whose essays are included in the analysis, are relatively young and well educated despite the application of purposive sampling. It cannot be ruled out that the results of our study are influenced by this, since it is known that health literacy is influenced by the patient's level of education and lifestyle commitments $[29,30]$. However, we included a highly variable sample of respondents and achieved saturation in the analysis. Moreover, we verified the figure and the position of the themes within it with the analysis of a second sample of essays. Therefore we feel confident that our results hold sufficient external validity.

\subsection{Conclusion}

The pathway to the diagnosis of PD is a dynamic and an iterative process. As described by patients, both patients and GPs have difficulties in recognizing the early symptoms of PD.

Patients often have a limited perception of PD, based on public figures with an advanced stage of PD. More than half of the patients believed they could have been diagnosed earlier. At the same time, the majority of all patients are content with their pathway. Nevertheless, patients can benefit a lot from an early diagnosis and timely therapeutic intervention, taking into account the patient's personal situation and preferences. Therefore it is important that patients, their significant others and GPs learn to recognize the early symptoms of PD and act accordingly.

\subsection{Practice implications}

In order to facilitate an earlier diagnosis of PD which enables shared decision making between patients and health care providers, educating the general population on possible prodromal symptoms of PD should be considered. The image of PD, as it is spread by the media, has to be modified from the classic image of the old man with advanced symptoms to a more complete representation of the disease.

It is necessary to explore whether the patient's view on possible lack of knowledge of the GP is underlined by the health care providers themselves. The GP has a central role in putting together all pieces of the puzzle which a patient has presented over time, in 
order to signal PD. Furthermore, (she)he is the gatekeeper to care, who has to refer to a neurologist. Therefore, it seems without debate that knowledge on the prodromal symptoms of $P D$ is essential. A subsequent quantitative analysis of all 900 collected essays of patients with PD is necessary and will give us more insight into the prodromal symptoms of PD that are experienced by patients and reported spontaneously by them.

Finally, we would strongly advise to no longer use 'stage(s) of delay' in the conversation concerning a diagnostic process. Delay has a negative connotation and does not appreciate the autonomy of the patient, who might decide to hold-back help seeking for a number of reasons. Furthermore, our results show that most patients are content with the pathway they experienced, although objectively spoken delay might have taken place. In other literature the term 'time interval' is suggested as an alternative for 'stage of delay' [21]. We believe this term accurately describes a stage (the time between two events) and, most importantly, does not criticize the decisions made by patients, alone or together with their health care providers.

\subsection{Ethics committee approval and informed consent}

The research ethics committee of the Radboud university medical center studied the protocol of the study and concluded that the study can be carried out in The Netherlands without an approval by the regional accredited research ethics committee (1112-2013).

The authors confirm that all personal identifiers have been removed or disguised so the patient(s) described are not identifiable and cannot be identified through the details of the story.

\section{Funding}

This study was supported by grants of the Dutch Parkinson's Disease Association 2012-V15 and the Health Insurers Innovation Foundation 2687.

\section{Acknowledgement}

The authors are grateful for the effort of the participating patient members of the Dutch Parkinson's Disease Association.

\section{References}

[1] Goldman JG, Postuma R. Premotor and nonmotor features of Parkinson's disease. Curr Opin Neurol 2014;27:434-41.

[2] Gaenslen A, Swid I, Liepelt-Scarfone I, Godau J, Berg D. The patients' perception of prodromal symptoms before the initial diagnosis of Parkinson's disease. Mov Disord 2011:26:653-8.

[3] Plouvier AO, Hameleers RJ, van den Heuvel EA, Bor HH, Olde Hartman TC, Bloem BR, et al. Prodromal symptoms and early detection of Parkinson's disease in general practice: a nested case-control study. Fam Pract 2014;31:373-8.
[4] Gonera EG, van't Hof M, Berger HJ, van Weel C, Horstink MW. Symptoms and duration of the prodromal phase in Parkinson's disease. Mov Disord 1997; 12:871-6.

[5] Pennebaker JW. The psychology of physical symptoms. New York: Springer; 1982.

[6] Gijsbers van Wijk CM, Kolk AM. Sex differences in perceived health. Ned Tijdschr Geneeskd 1997;141:283-7.

[7] Cameron L, Leventhal EA, Leventhal H. Symptom representations and affect as determinants of care seeking in a community-dwelling, adult sample population. Health Psychol 1993;12:171.

[8] Evans D, Norman P. Illness representations, coping and psychological adjustment to Parkinson's disease. Psychol Health 2009;24:1181-96.

[9] Breen DP, Evans JR, Farrell K, Brayne C, Barker RA. Determinants of delayed diagnosis in Parkinson's disease. J Neurol 2013;260:1978-81.

[10] Weerkamp NJ, Nijhof A, Tissingh G. Non-motor symptoms of Parkinson's disease. Ned Tijdschr Geneeskd 2012:156:A3926.

[11] Saunders-Pullman R, Wang C, Stanley K, Bressman SB. Diagnosis and referral delay in women with Parkinson's disease. Gend Med 2011;8:209-17.

[12] Rana AQ, Siddiqui I, Yousuf MS. Challenges in diagnosis of young onset Parkinson's disease. J Neurol Sci 2012;323:113-6.

[13] Lohle M, Ramberg CJ, Reichmann H, Schapira AH. Early versus delayed initiation of pharmacotherapy in Parkinson's disease. Drugs 2014;74:645-57.

[14] Mestre TA, Teodoro T, Reginold W, Graf J, Kasten M, Sale J, et al. Reluctance to start medication for Parkinson's disease: a mutual misunderstanding by patients and physicians. Parkinsonism Relat Disord 2014;20:608-12.

[15] Grosset D, Taurah L, Burn DJ, MacMahon D, Forbes A, Turner K, et al. A multicentre longitudinal observational study of changes in self reported health status in people with Parkinson's disease left untreated at diagnosis. J Neurol Neurosurg Psychiatry 2007;78:465-9.

[16] de Nooijer J, Lechner L, de Vries H. A qualitative study on detecting cancer symptoms and seeking medical help; an application of Andersen's model of total patient delay. Patient Educ Couns 2001;42:145-57.

[17] Usher-Smith JA, Thompson MJ, Walter FM. Looking for the needle in the haystack': a qualitative study of the pathway to diagnosis of type 1 diabetes in children. BMJ Open 2013;3:e004068.

[18] Elo S, Kyngas H. The qualitative content analysis process. J Adv Nurs 2008:62:107-15

[19] Safer MA, Tharps QJ, Jackson TC, Leventhal H. Determinants of three stages of delay in seeking care at a medical clinic. Med Care 1979;17:11-29.

[20] Andersen BL, Cacioppo JT. Delay in seeking a cancer diagnosis: delay stages and psychophysiological comparison processes. Br J Soc Psychol 1995;34(Pt 1):33-52.

[21] Walter F, Webster A, Scott S, Emery J. The Andersen Model of Total Patient Delay: a systematic review of its application in cancer diagnosis. J Health Serv Res Policy 2012;17:110-8.

[22] Smith LK, Pope C, Botha JL. Patients' help-seeking experiences and delay in cancer presentation: a qualitative synthesis. Lancet 2005;366:825-31.

[23] Leydon GM, Bynoe-Sutherland J, Coleman MP. The journey towards a cancer diagnosis: the experiences of people with cancer, their family and carers. Eur J Cancer Care (Engl) 2003;12:317-26.

[24] Miller WR, Buelow JM, Bakas T. Older adults and new-onset epilepsy: experiences with diagnosis. J Neurosci Nurs 2014;46:2-10.

[25] Walter U, Kleinschmidt S, Rimmele F, Wunderlich C, Gemende I, Benecke R, et al. Potential impact of self-perceived prodromal symptoms on the early diagnosis of Parkinson's disease. J Neurol 2013:260:3077-85.

[26] Bloem BR, Stocchi F. Move for change part I: A European survey evaluating the impact of the EPDA Charter for People with Parkinson's disease. Eur J Neurol 2012:19:402-10.

[27] Joseph-Williams N, Elwyn G, Edwards A. Knowledge is not power for patients: a systematic review and thematic synthesis of patient-reported barriers and facilitators to shared decision making. Patient Educ Couns 2014:94:291-309.

[28] Frosch DL, May SG, Rendle KA, Tietbohl C, Elwyn G. Authoritarian physicians and patients' fear of being labeled 'difficult' among key obstacles to shared decision making. Health Aff (Millwood) 2012;31:1030-8.

[29] Smith SK, Dixon A, Trevena L, Nutbeam D, McCaffery KJ. Exploring patient involvement in healthcare decision making across different education and functional health literacy groups. Soc Sci Med 2009;69:1805-12.

[30] Jordan JE, Buchbinder R, Osborne RH. Conceptualising health literacy from the patient perspective. Patient Educ Couns 2010;79:36-42. 